\title{
ABOUT THE EDGE-BASED SMOOTHED FINITE ELEMENT METHOD FOR THE REISSNER-MINDLIN PLATE-BENDING PROBLEM
}

\author{
Nguyen Xuan Hung ${ }^{1,2}$, Nguyen Thoi Trung ${ }^{1,3}$ \\ ${ }^{1}$ Department of Mechanics, Faculty of Mathematics and Computer Science, \\ University of Science-VNU-HCM, Vietnam \\ ${ }^{2}$ Singapore-MIT Alliance (SMA), E4-04-10, 4 Engineering Drive 3, Singapore \\ ${ }^{3}$ Center for Advanced Computations in Engineering Science (ACES), \\ Department of Mechanical Engineering, National University of Singapore, \\ 9 Engineering Drive 1, Singapore
}

\begin{abstract}
The paper further develops the edge-based smoothed finite element method (ES-FEM) for analysis of Reissner - Mindlin plates using triangular meshes. The bending and shearing stiffness matrices are obtained using strain smoothing technique over the smoothing domains associated with edges of elements. Transverse shear locking can be avoided with help of the discrete shear gap (DSG) method. The numerical examples show that the present ES-FEM-DSG method obtains very accurate results compared to the exact solution and other existing elements.
\end{abstract}

\section{INTRODUCTION}

In the practical applications, lower-order plate finite elements are the most preferred due to its simplicity and efficiency. However, using the Reissner-Mindlin plate theory, these elements often suffer from one intrinsic difficulty: shear locking phenomenon in the limitation of thin plates. In order to eliminate shear locking, early methods tried to avoid shear locking by using reduced integration or a selective reduced integration [1]. For example, based on a four node quadrilateral element, a single Gauss point is utilized to compute shear strain energy while a 2x2 Gauss point scheme is used for the bending energy. Unfortunately, reduced integration often causes the instability due to rank deficiency of and results in zero-energy modes [1]. Various improvements of formulations as well as numerical techniques have been developed such as mixed formulation/hybrid elements [2], Enhanced Assumed Strain (EAS) method [3] or Assumed Natural Strain (ANS) method [4,5]. An alternative to the ANS method to avoid shear locking is the Discrete-Shear-Gap (DSG) method [6]. The DSG method is in a way similar to the ANS method since it modifies the course of certain strains within the element, but the difference in the aspect of the lack of collocation points that permits the DSG method independent of the order and form of the element.

Recently, Liu et al. have incorporated a strain smoothing technique [7] with the FEM to formulate a cell/element-based smoothed finite element method (SFEM or CS-FEM) [8,9]. CS-FEM is based on the smoothing domains located inside the elements and proposed to 
solve $2 \mathrm{D}$ solid mechanics problems $[8,9,10,11,12]$. The essence and the evaluation of the shape functions for the SFEM has been further investigated in [13]. CS-FEM has then been extended to plate and shell structures $[14,15,16]$ and fracture mechanics problems [17]. A node-based smoothed finite element method (NS-FEM) [18] was recently formulated to produce upper bound solutions in the strain energy and also eliminates volumetric locking naturally. However, the NS-FEM was found overly soft temporally instable, and can not applied directly to dynamic problems. To overcome such a temporal instability, Liu et al [19] have lately proposed an edge-based smoothed finite element method (ES-FEM) to significantly improve the accuracy and convergence rate of the standard finite element formulation for static, free and forced vibration analyses of solids using triangular elements. It has been demonstrated that the ES-FEM using triangular meshes is always stable, efficient, and even more accurate than the standard FEM using quadrilateral elements (Q4) without any additional degrees of freedom. The ES-FEM was then developed for analysis of static and eigenvalue of two-dimensional piezoelectric structures [20] and dynamic analyses of plates [21].

This paper further develops the ES-FEM triangular element for static analysis of Reissner-Mindlin plates. The bending and shearing stiffness matrices are obtained using strain smoothing technique over the smoothing domains associated with edges of elements. Transverse shear locking can be avoided with help of the discrete shear gap (DSG) method. The numerical examples show that the present ES-FEM-DSG method obtains very accurate results compared to the exact solution and other existing elements.

\section{GOVERNING EQUATIONS AND WEAK FORM}

Let $\Omega$ be the region in $R^{2}$ occupied by the middle plane of the plate. $w$ and $\beta=$ $\left(\beta_{x}, \beta_{y}\right)^{T}$ denote the transverse displacement and the rotations in the $\mathrm{X}-\mathrm{z}$ and $\mathrm{y}-\mathrm{z}$ planes, cf. Figure 1, respectively. Assuming that the material is homogeneous and isotropic with Young's modulus $E$ and Poisson ratio $\nu$, the governing differential equations of ReissnerMindlin plate are of the form,

$$
\begin{gathered}
\operatorname{div} \mathbf{D}^{b} \boldsymbol{\kappa}(\boldsymbol{\beta})+\lambda t \gamma(\boldsymbol{\beta})=0 \text { in } \Omega \\
-\lambda t \operatorname{div}(\boldsymbol{\gamma})=p \text { in } \Omega \\
w=\bar{w}, \boldsymbol{\beta}=\overline{\boldsymbol{\beta}} \text { on } \Gamma=\partial \Omega
\end{gathered}
$$

where $t$ is the plate thickness, $p=p(x, y)$ is the transverse loading per unit area, $\lambda=\frac{k E}{2(1+\nu)}$, $k=5 / 6$ is the shear correction factor and $\mathbf{D}^{b}$ is the tensor of bending module, $\kappa$ and $\gamma$ are the bending and shear strains, respectively, defined by

$$
\boldsymbol{\kappa}=\left[\begin{array}{c}
\frac{\partial \beta_{x}}{\partial x} \\
-\frac{\partial \beta_{y}}{\partial y} \\
\frac{\partial \beta_{x}}{\partial y}-\frac{\partial \beta_{y}}{\partial x}
\end{array}\right], \gamma=\left[\begin{array}{c}
\frac{\partial w}{\partial x}+\beta_{x} \\
\frac{\partial w}{\partial y}-\beta_{y}
\end{array}\right]
$$

The Eqs. (1) - (3) correspond to the minimization of the total potential function

$$
\Pi=\frac{1}{2} \int_{\Omega} \kappa^{T} \mathbf{D}^{b} \boldsymbol{\kappa} d \Omega+\frac{1}{2} \int_{\Omega} \gamma^{T} \mathbf{D}^{s} \gamma d \Omega-\int_{\Omega} w p d \Omega
$$




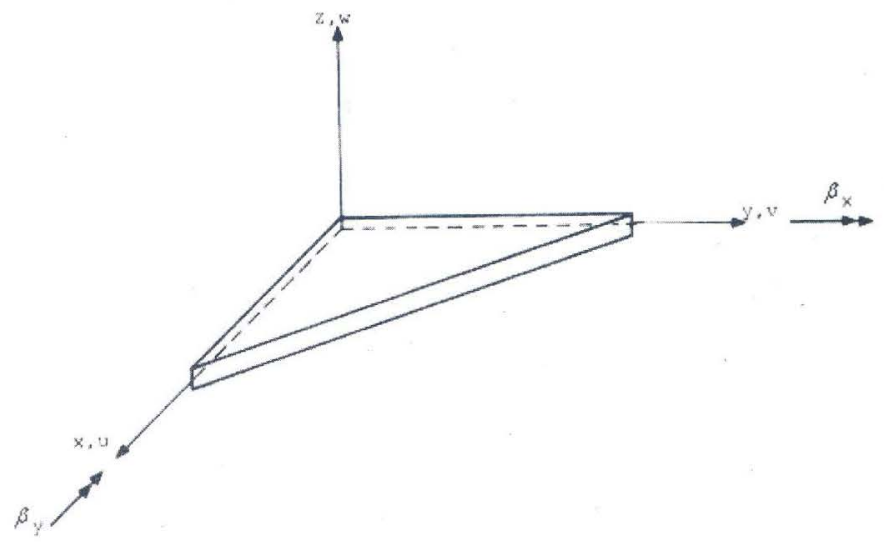

Fig. 1. Three-node triangular element and definition of the rotations

Assumed that the bounded domain $\Omega$ is discretized into $n_{e}$ finite elements, $\Omega \approx \Omega^{h}=$ $\sum_{e=1}^{n_{e}} \Omega^{e}$. The finite element solution $\mathbf{u}^{h}=\left[w \beta_{x} \beta_{y}\right]^{T}$ of a displacement model for ReissnerMindlin plate is expressed as follows

$$
\mathbf{u}^{h}=\sum_{i=1}^{n p}\left[\begin{array}{ccc}
N_{I} & 0 & 0 \\
0 & 0 & N_{I} \\
0 & N_{I} & 0
\end{array}\right] \mathbf{q}_{I}
$$

where $n p$ is total the number of element nodes, the $N_{I}$ 's are the bilinear shape interpolation functions associated to node $I$, the $\mathbf{q}_{I}=\left[\begin{array}{lll}w_{I} & \theta_{x I} & \theta_{y I}\end{array}\right]^{T}$ are the nodal degrees of freedom of the variables $\mathbf{u}^{h}=\left[\begin{array}{lll}w & \beta_{x} & \beta_{y}\end{array}\right]^{T}$ associated to node $I$. Then, the discrete curvature and shear fields are

$$
\kappa^{h}=\mathbf{B}^{b} \mathbf{q}, \gamma^{h}=\mathbf{B}^{s} \mathbf{q}
$$

where

$$
\mathbf{B}_{I}^{b}=\left[\begin{array}{ccc}
0 & 0 & N_{I, x} \\
0 & -N_{I, y} & 0 \\
0 & -N_{I, x} & N_{I, y}
\end{array}\right], \mathbf{B}_{i}^{s}=\left[\begin{array}{ccc}
N_{i, x} & 0 & N_{i} \\
N_{i, y} & -N_{i} & 0
\end{array}\right]
$$

By substituting Eqs. (6) - (8) into Eq. (5) and minimization, we obtain a linear system of equations for the vector of nodal unknowns $q$,

$$
\mathbf{K q}=\mathbf{g}
$$

with the global stiffness matrix given by

$$
\mathbf{K}=\int_{\Omega}\left(\mathbf{B}^{b}\right)^{T} \mathbf{D}^{b} \mathbf{B}^{b} d \Omega+\int_{\Omega}\left(\mathbf{B}^{s}\right)^{T} \mathbf{D}^{s} \mathbf{B}^{s} d \Omega
$$


and the load vector by

$$
\mathbf{g}_{i}=\int_{\Omega} N_{i}\left[\begin{array}{l}
p \\
0 \\
0
\end{array}\right] d \Omega
$$

where

$$
\mathbf{D}^{b}=\frac{E t^{3}}{12\left(1-\nu^{2}\right)}\left[\begin{array}{ccc}
1 & \nu & 0 \\
\nu & 1 & 0 \\
0 & 0 & \frac{1-\nu}{2}
\end{array}\right], \mathbf{D}^{s}=\lambda t\left[\begin{array}{ll}
1 & 0 \\
0 & 1
\end{array}\right]
$$

As already mentioned in the introduction, for a low-order element such as three triangular element, shear locking often occurs when the thickness of plates reduces to zero. Therefore, there are various techniques for eliminating locking found in the literatures [1].

In this paper, we introduce a simple triangular plate element that combines two following important issues: 1) The edge-based smoothed finite element method (ES-FEM) that was found to be one of the "most" accurate models using triangular elements, 2) A Discrete Shear Gap (DSG) concept for shear-locking-free triangular Reissner-Mindlin plate-bending finite element (DSG3).

For reference, the edge-based smoothed finite element method (ES-FEM) with discrete shear gap (DSG) technique using triangular elements is also termed as ES-DSG3 for short.

\section{AN ES-FEM WITH DISCRETE SHEAR TECHNIQUE}

We start the approximation $\mathbf{u}^{h}=\left[\begin{array}{lll}w & \beta_{x} & \beta_{y}\end{array}\right]^{T}$ of three-node triangular element. The finite Reissner-Mindlin plate-bending element approximation is simply interpolated using the linear basis functions for both deflection and rotations without any additional variables. Hence, the bending strains are constant and unchanged from the standard finite elements while the transverse shear strains contain linear interpolated functions. Applying the discrete shear gaps (DSG) [6], the shear strains $\gamma^{h}$ become constant and aim to avoid shear locking problem.

In the ES-FEM, we do not use the compatible strain fields as in the standard FEM but strains "smoothed" over local smoothing domains, and naturally the integration for the stiffness matrix is no longer based on elements, but these smoothing domains. These local smoothing domains are constructed based on edges of the elements such that $\Omega \approx$ $\Omega^{h}=\sum_{k=1}^{N_{e}} \Omega^{(k)}$ and $\Omega^{(i)} \cap \Omega^{(k)} \neq \emptyset$ for $i \neq j$, in which is the total number of edges of all elements in the entire problem domain. For triangular elements, the smoothing domain $\Omega^{(k)}$ associated with the edge $k$ is created by connecting two end-nodes of the edge to centroids of adjacent elements as shown in Figure 2.

Introducing smoothing curvature, shear strain over the smoothing domain $\Omega^{(k)}$, one has

$$
\tilde{\boldsymbol{\kappa}}_{k}=\int_{\Omega^{(k)}} \kappa^{h}(\mathbf{x}) \Phi(\mathbf{x}) d \Omega, \tilde{\gamma}_{k}=\int_{\Omega^{(k)}} \gamma^{h}(\mathbf{x}) \Phi(\mathbf{x}) d \Omega
$$

where $\Phi$ is a smoothing function that generally satisfies the following properties [7]

$$
\Phi \geq 0 \text { and } \int_{\Omega^{h}} \Phi(\mathbf{x}) d \Omega=1
$$


For simplicity, $\Phi$ is assumed to be a step function defined by

$$
\Phi(\mathbf{x})=\left\{\begin{array}{l}
1^{z} / A^{(k)}, \mathbf{x} \in \Omega^{(k)} \\
0, \mathbf{x} \notin \Omega^{(k)}
\end{array}\right.
$$

where $A^{(k)}$ is the area of the smoothing domain and is computed by

$$
A^{(k)}=\frac{1}{3} \sum_{i=1}^{N_{e}^{k}} A_{i}
$$

where $N_{e}^{k}$ is the number of elements containing the edge $k\left(N_{e}^{k}=1\right.$ for the boundary edges and $N_{e}^{k}=2$ for inner edges as shown in Figure 2) and $A_{i}$ is the area of the $i$ th element around the edge $k$. Substituting Eq. (15) into Eq. (13), the average strains at edge $k$ can

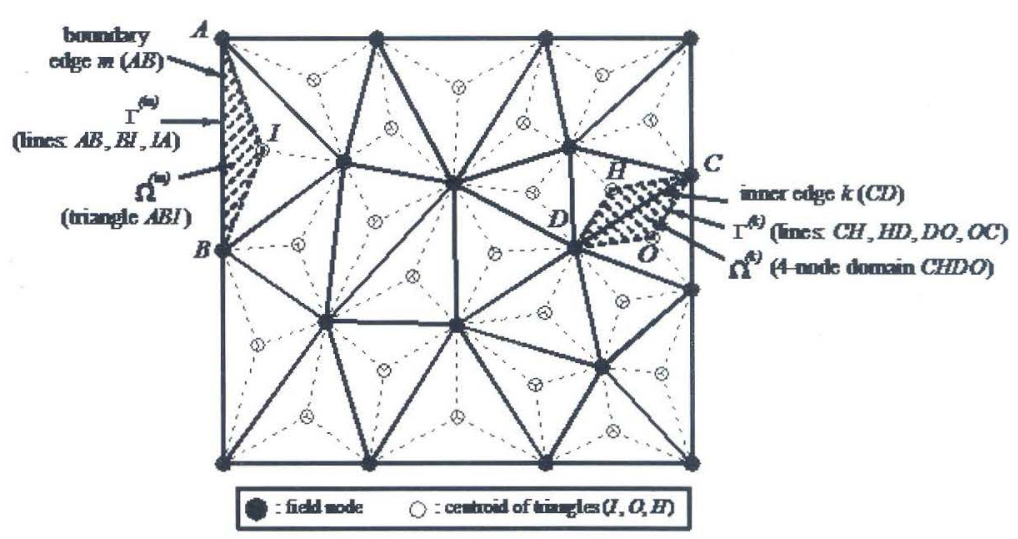

Fig. 2. Division of domain into triangular element and smoothing domains $\Omega^{(k)}$ connected to edge $k$ of triangular elements.

be expressed in the following form

$$
\tilde{\boldsymbol{\kappa}}_{k}=\frac{1}{A^{(k)}} \int_{\Omega^{(k)}} \boldsymbol{\kappa}^{h}(\mathbf{x}) d \Omega=\sum_{I=1}^{N_{n}^{k}} \tilde{\mathbf{B}}_{I}^{b} \mathbf{q}_{I}, \tilde{\gamma}_{k}=\frac{1}{A^{(k)}} \int_{\Omega^{(k)}} \gamma^{h}(\mathbf{x}) d \Omega=\sum_{I=1}^{N_{n}^{k}} \tilde{\mathbf{B}}_{I}^{s} \mathbf{q}_{I}
$$

where $N_{n}^{k}$ is the number of nodes belonging to elements directly connected to edge $k$ $\left(N_{n}^{k}=3\right.$ for boundary edges and $N_{n}^{k}=4$ for inner edges as shown in Figure 2) and $\tilde{\mathbf{B}}_{I}^{b}$, $\tilde{\mathbf{B}}_{I}^{s}$ are the average gradient matrices through the smoothing domain $\Omega^{(k)}$ and given by

$$
\tilde{\mathbf{B}}_{I}^{b}=\frac{1}{A^{(k)}} \sum_{i=1}^{N_{e}^{k}} \frac{1}{3} A_{i} \mathbf{B}_{i}^{b}, \tilde{\mathbf{B}}_{I}^{s}=\frac{1}{A^{(k)}} \sum_{i=1}^{N_{e}^{k}} \frac{1}{3} A_{i} \mathbf{B}_{i}^{s}
$$


where $\mathbf{B}_{i}^{b}$ (of $3 \times 3$ constant matrix) is obtained from the three-node standard finite element,

$$
\tilde{\mathbf{B}}_{i}^{b}=\frac{1}{2 A_{e}}\left(\begin{array}{ccccccccc}
0 & b-c & 0 & 0 & c & 0 & 0 & -b & 0 \\
0 & 0 & d-a & 0 & 0 & -d & 0 & 0 & a \\
0 & d-a & b-c & 0 & -d & c & 0 & a & -b
\end{array}\right)
$$

while $\mathbf{B}_{i}^{s}$ (of $2 \times 3$ constant matrix) is derived from the discrete shear gap technique

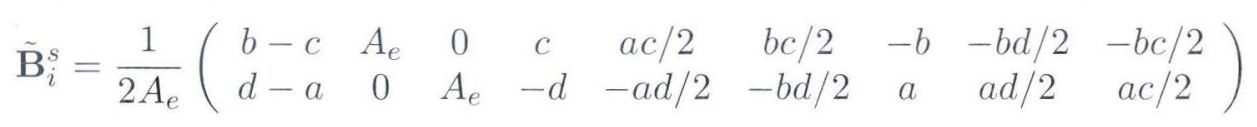

with $a=x_{2}-x_{1}, b=y_{2}-y_{1}, c=y_{3}-y_{1}, d=x_{3}-x_{1}$ and $A_{e}$ is the area of the triangular element.

The global stiffness matrix of the ES-DSG3 element is assembled by the following form

$$
\tilde{\mathbf{K}}=\sum_{k=1}^{N_{e}} \tilde{\mathbf{K}}^{(k)}
$$

where $\tilde{\mathbf{K}}^{(k)}$ is the edge stiffness matrix given by

$$
\tilde{\mathbf{K}}^{(k)}=\int_{\Omega^{(k)}} \tilde{\mathbf{B}}^{b T} \mathbf{D}^{b} \tilde{\mathbf{B}}^{b} d \Omega+\int_{\Omega^{(k)}} \tilde{\mathbf{B}}^{s T} \mathbf{D}^{s} \tilde{\mathbf{B}}^{s} d \Omega=\tilde{\mathbf{B}}^{b T} \mathbf{D}^{b} \tilde{\mathbf{B}}^{b} A^{(k)}+\tilde{\mathbf{B}}^{s T} \mathbf{D}^{s} \tilde{\mathbf{B}}^{s} A^{(k)}
$$

It is seen from Eq. (22) that the stiffness matrix is analytically computed from the integrated constant matrices. Note that the rank of the ES-DSG3 element is similar to that of the DSG3 element and the stability of the ES-DSG3 element is also ensured.

\section{NUMERICAL RESULTS}

In this section, benchmark problems are examined for the present method. For comparison. several published elements mentioned in this paper are denoted as follows:

- MIN3 - Tessler's anisoparametric Reissner/Mindlin plate 3-node triangular element [4].

- MITC4 - The mixed interpolation of tensorial components for four-node plate element [5].

- DSG3 - The Discrete Shear Gap triangular element [6].

- ES-DSG3 - The edge-based smoothed discrete shear gap triangular element that is presented in this paper. More details for dynamics analyses using the ES-DSG3 can be found in [21].

In addition, other published models appeared in the context of the paper are also correspondingly cited if they are not yet abbreviated above.

\subsection{Patch test}

First we investigate the element behavior with the patch test. This is a numerical technique to prove that the proposed method will or will not converge. A plate with four triangular elements is shown in Figure 3. The boundary deflection is assumed to be $w=\frac{1}{2}\left(1+x+2 y+x^{2}+x y+y^{2}\right)$. The results given in Table 1 enjoy that all the MIN3, DSG3 and ES-DSG3 elements pass the patch test with machine precision. 


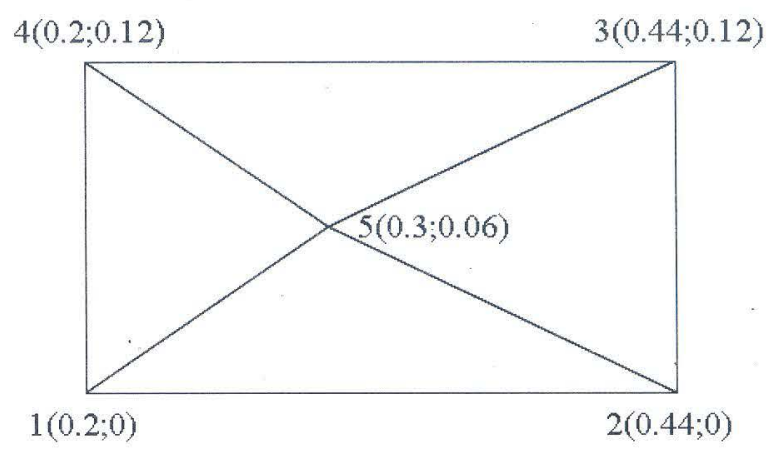

Fig. 3. Patch test of elements

Table 1. Patch test

\begin{tabular}{ccccccc}
\hline Element & $w_{5}$ & $\theta_{x 5}$ & $\theta_{y 5}$ & $m_{x 5}$ & $m_{y 5}$ & $m_{x y 5}$ \\
\hline MIN3 & 0.6422 & 1.1300 & -0.6400 & -0.01111 & -0.01111 & -0.00333 \\
DSG3 & 0.6422 & 1.1300 & -0.6400 & -0.01111 & -0.01111 & -0.00333 \\
ES-DSG3 & 0.6422 & 1.1300 & -0.6400 & -0.01111 & -0.01111 & -0.00333 \\
Exact & 0.6422 & 1.1300 & -0.6400 & -0.01111 & -0.01111 & -0.00333 \\
\hline
\end{tabular}

\subsection{Square plates}

Various square plate models subjected to a uniform load are described in Figure 4 . The geometry and material parameters are given as length $L=10$, thickness $t=0.1$, Young's modulus $E=1092000$, Poisson's ratio $\nu=0.3$ and $p=1$. The plates with the symmetry and uniform mesh $N \times N(N=2,4,8,16,32)$ are modeled as in the lower left corner of Figure 4.

Now, we assess the performance of ES-DSG3 element compared with several other elements such as MIN3, MITC4 and DSG3 elements. Figure 5 shows the convergence of the normalized deflection and the normalized moment at the center of the simply supported plate against the mesh density $N$. It is seen that the ES-DSG3 element achieves higher accuracy than the DSG3 and MIN3 elements. For the convergence of the central deflection, the MITC4 element is the most effective due to using the bilinear function approximations. For the convergence of moment with fine meshes, the ES-DSG3 element is slightly more accurate than the MITC4 element.

For a clamped plate, the convergence of the normalized deflection and the normalized moment at the center is shown in Figure 6. It is clear that the present element is free of shear locking in the limit of the thin plate. In addition, the ES-DSG3 achieves the higher accuracy than the DSG3 and MIN3 elements. For very coarse meshes, the fournode MITC4 plate element is more accurate than the ES-DSG3 element. However, the ES-DSG3 element is slightly more accurate than the MITC4 element for finer meshes. 


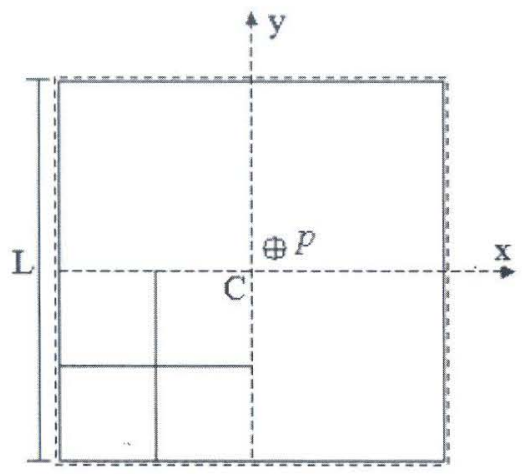

(a)

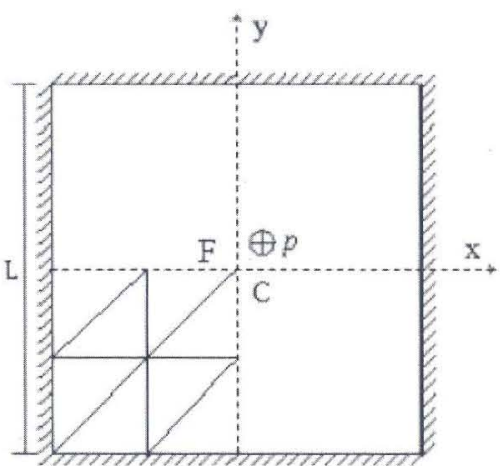

(b)

Fig. 4. Square plate model subjected to a uniformly distributed load $p$ : (a) simply supported condition; (b) full clamped condition

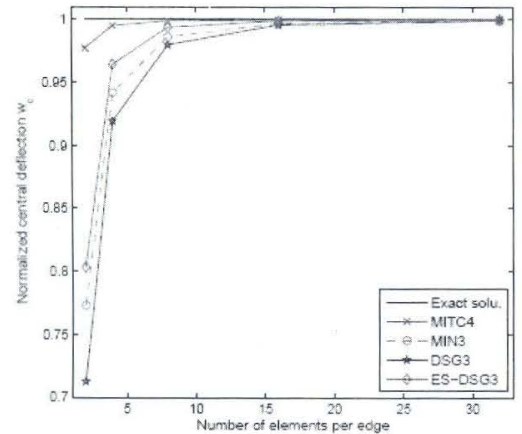

(a)

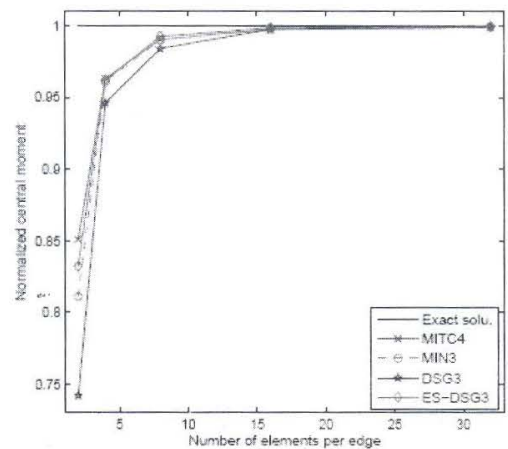

(b)

Fig. 5. Normalized deflection and moment at center of simply support square plate subjected to uniform load

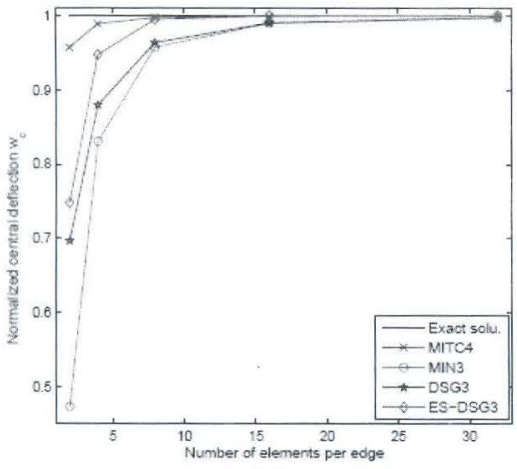

(a)

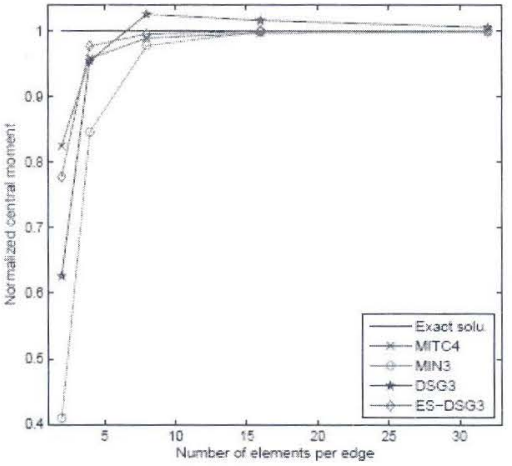

(b)

Fig. 6. Normalized deflection and moment at center of fully clamped square plate subjected to uniform load 


\subsection{Morley's skew rhombic plate}

A rhombic plate with skew angle $30^{0}$ simply supported $\operatorname{soft}(w=0)$ on all edges and subjected to a uniform load $p=1$. Problem model and initial mesh with $4 \times 4$ elements are illustrated in Figure 7a. Data is given as follows: $L=10, t=0.1, E=10.92, \nu=$ 0.3 . This benchmark problem which was originally mentioned by Morley [22] occurs the high singularity at two obtuse corners of the plate, see Figure 8. It is clear seen from Figure 9 that the ES-DSG3 element shows remarkably excellent performance compared to the MIN3, DSG3, MITC4 elements and a large number of other published elements reported in [23].

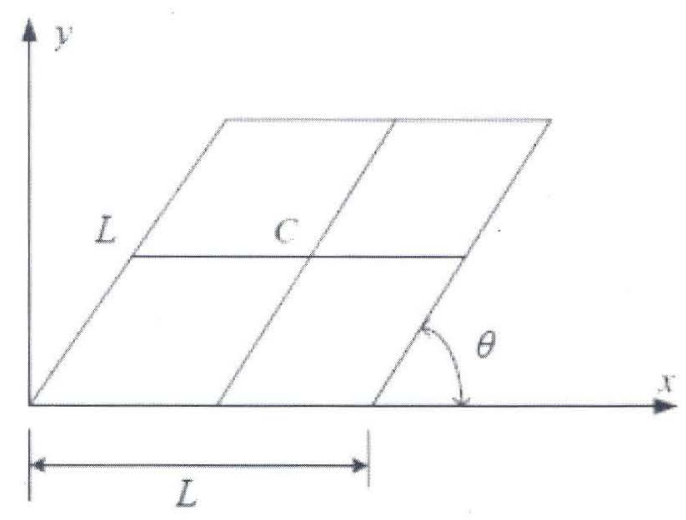

(a)

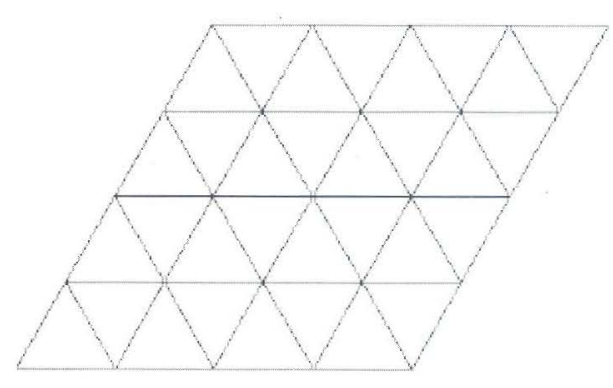

(b)

Fig. 7. A simply supported skew plate subjected to a uniform load

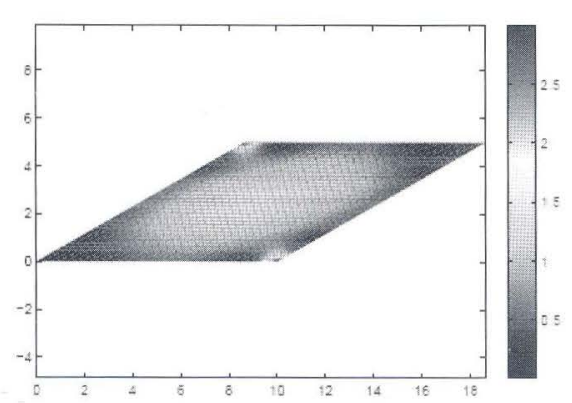

(a)

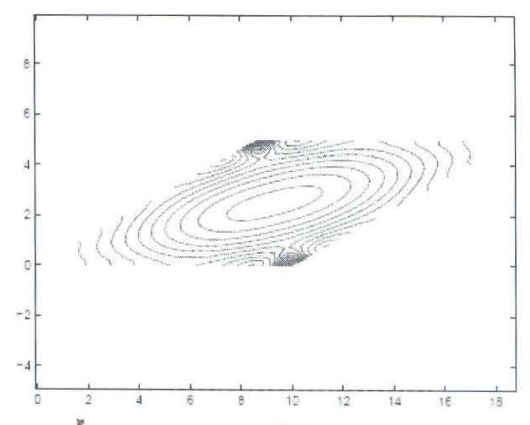

(b)

Fig. 8. A distribution of von Mises and level lines for skew plate using ES-DSG3 element 


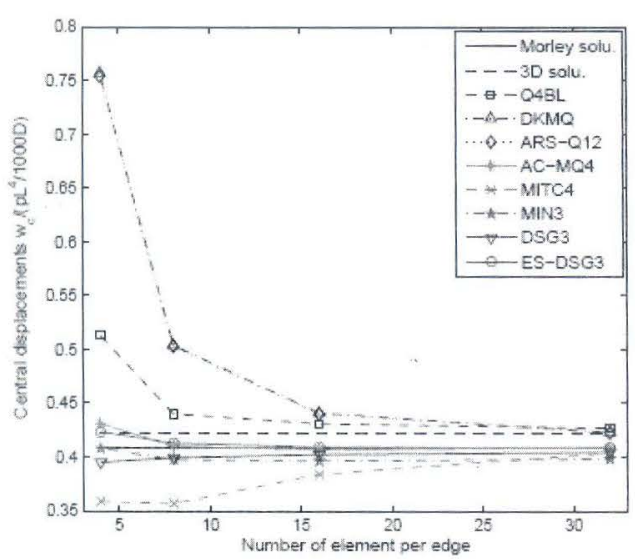

(a)

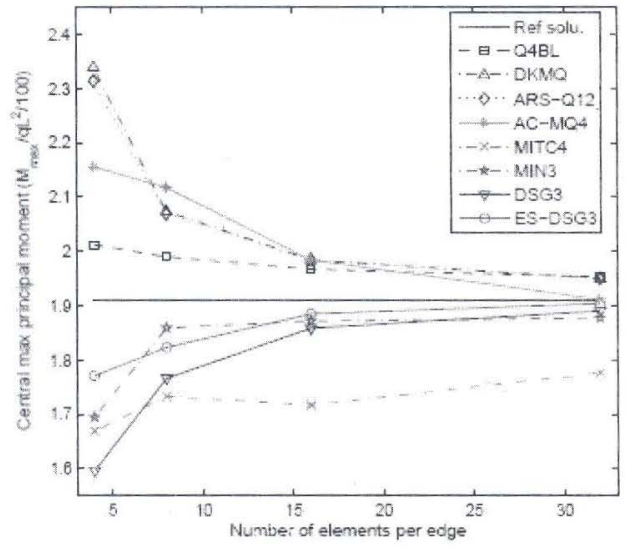

(b)

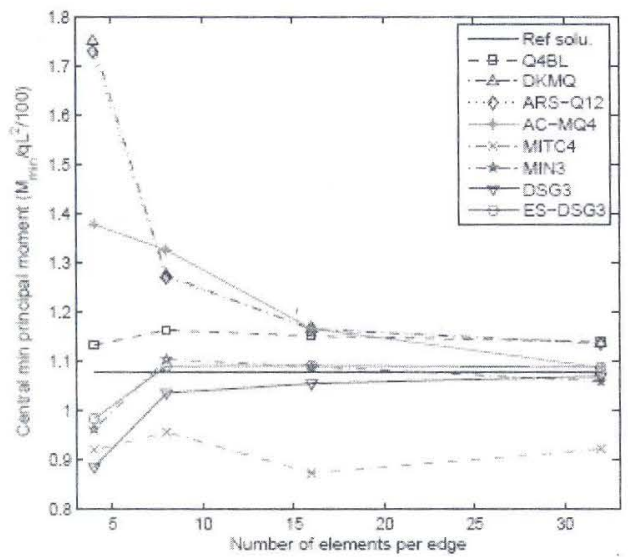

(c)

Fig. 9. Normalized deflection and moment at center of fully clamped square plate subjected to uniform load

\section{CONCLUSION}

This paper shows the numerical performances of the ES-FEM (ES-DSG3 element) for Reissner-Mindlin plates. The element formulations only uses triangular meshes and are simply obtained based on the bending and shear strains smoothed over the smoothing domain associated with the edges of the triangles. The ES-DSG3 uses only three DOFs at each vertex node without additional degrees of freedom and no more requirement of high computational cost.

The ES-DSG3 element eliminates well shear locking phenomena and more accurate than the DSG3, MIN3 triangular elements when the same sets of nodes are used. The results of the ES-DSG3 element are also in a good agreement with analytical solution and show remarkably excellent performance compared to results of several other published 
triangular-quadrilateral elements in the literature. It has been observed that ES-DSG3 element performs excellently the skew plate with strong singularity at the obtuse corners.

\section{REFERENCES}

[1] O.C. Zienkiewicz and R.L. Taylor, The Finite Element Method, 5th Edition, Butterworth Heinemann(Oxford) (2000).

[2] T.H. H. Pian, Hybrid and incompatible finite element method, CRC Press: Boca Raton (FL) (2006).

[3] J.C. Simo and M.S. Rifai, A class of mixed assumed strain methods and the method of incompatible modes, International Journal for Numerical Methods in Engineering 29 (1990) 1595-1638.

[4] A. Tessler, T. J. R. Hughes, A three-node Mindlin plate element with improved Transverse Shear, Computer methods in Applied Mechanics and Engineering 50 (1985) 71101.

[5] K.J. Bathe and E.N. Dvorkin, A four-node plate bending element based on MindlinReissener plate theory and a mixed interpolation, International Journal for Numerical Methods in Engineering 21 (1985) 367 - 383.

[6] K.U. Bletzinger, M. Bischoff, E. Ramm, A unified approach for shear-locking free triangular and rectangular shell finite elements, Computers and Structures $\mathbf{7 5}$ (2000) 321-334.

[7] J.S. Chen, C.T. Wu, S. Yoon and Y. You, A stabilized conforming nodal integration for Galerkin mesh-free methods, International Journal for Numerical Methods in Engineering 50(2001) 435-466.

[8] G.R. Liu, K.Y. Dai and T.T. Nguyen, A smoothed finite element for mechanics problems, Computational Mechanics 39 (2007) 859-877.

[9] G.R. Liu, T.T. Nguyen, K.Y. Dai and K.Y. Lam, Theoretical aspects of the smoothed finite element method (SFEM), International Journal for Numerical Methods in Engineering 71 (2007) 902-930.

[10] K.Y. Dai, G.R. Liu, T.T. Nguyen, An n-sided polygonal smoothed finite element method (nSFEM) for solid mechanics, Finite Element Analysis and Design 43 (2007) $847-860$.

[11] K.Y. Dai, G.R. Liu, Free and forced vibration analysis using the smoothed finite element method (SFEM), Journal of Sound and Vibration 301 (2007) $803-820$.

[12] H. Nguyen-Xuan, S. Bordas and H. Nguyen-Dang, Smooth finite element methods: Convergence, accuracy and properties, International Journal for Numerical Methods in Engineering 301 (2008) 175-208.

[13] G.R. Liu, T.Nguyen-Thoi, H. Nguyen-Xuan, K. Y. Dai, K. Y. Lam, On the essence and the evaluation of the shape functions for the smoothed finite element method (SFEM), International Journal for Numerical Methods in Engineering 77 (2009) 1863-1869

[14] H. Nguyen-Xuan, T. Rabczuk, S. Bordas and J.F. Debongnie, A smoothed finite element method for plate analysis, Computer methods in Applied Mechanics and Engineering 197 (2008) 1184-1203. 
[15] N. Nguyen-Thanh, T. Rabczuk, H. Nguyen-Xuan and S. Bordas, A smoothed finite element method for shell analysis, Computer methods in Applied Mechanics and Engineering 198 (2008) 165-177.

[16] H. Nguyen-Xuan, T. Nguyen-Thoi, A stabilized smoothed finite element method for free vibration analysis of Mindlin-Reissner plates, Communication in Numerical Methods in Engineering in press (2008) doi: 10.1002/cnm.1137.

[17] S. Bordas, T. Rabczuk, H. Nguyen-Xuan, P. Nguyen Vinh, S. Natarajan,T. Bog, Q. Do Minh,H. Nguyen Vinh, Strain smoothing in FEM and XFEM, Computers and Structures in press (2008) doi:10.1016/j.compstruc.2008.07.006.

[18] G.R. Liu, T. Nguyen-Thoi, H. Nguyen-Xuan, K.Y. Lam, A node-based smoothed finite element method (NS-FEM) for upper bound solutions to solid mechanics problems, Computers and Structures 87 (2009) 14-26.

[19] G.R. Liu, T. Nguyen-Thoi, K.Y. Lam, An edge-based smoothed finite element method (ES-FEM) for static, free and forced vibration analyses of solids, Journal of Sound and Vibration 320 (2009) 1100-1130.

[20] H. Nguyen-Xuan, G.R. Liu, T. Nguyen-Thoi and C. Nguyen-Tran, An edge-based smoothed finite element method (ES-FEM) for static, free and forced vibration analyses of solids, Smart Material and Structures 18 (2009) 065015 (12pp).

[21] H. Nguyen-Xuan, GR Liu, C. Thai-Hoang, T. Nguyen-Thoi, An edge-based smoothed finite element method with stabilized discrete shear gap technique for analysis of Reissner-Mindlin plates, Computer Methods Applied Mechanics Engineering (2009) in press, doi:10.1016/j.cma.2009.09.001.

[22] L.S.D. Morley, Skew plates and structures, Pergamon Press: Oxford (1963).

[23] S. Cen, Y.Q. Long, Z.H. Yao and S.P. Chiew, Application of the quadrilateral area co-ordinate method:A new element for Mindlin-Reissner plate, International Journal for Numerical Methods in Engineering 66(2006) 1 - 45.

Received May 14, 2009

\section{PHƯƠNG PHÁP PHẦN TỬ HỮU HAN TRƠN DỰA TRÊN CANH CHO BÀI TOÁN REISSNER - MINDLIN}

Bài báo này phát triển mô tả phương pháp phần tử hữu hạn trơn dựa trên cạnh cho bài toán uốn tấm Reissner-Mindlin. Các ma trận độ cứng uốn và cắt đạt được dùng kỹ thuật trơn hóa biến dạng qua miền trơn được kết hợp với cạnh của phần tử. Hiện tượng "shear locking" có thể khác phục ding phương pháp rời rạc lệch trượt (DSG). Các ví dụ số cho thấy phương pháp ES-FEM-DSG đạt được kết quả rất chính xác so với nghiệm giải tích và nhiều phần tử đã được công bố. 